\title{
Buried Hydrogen Bond Interactions Contribute to the High Potency of Complement Factor D Inhibitors
}

Chao-Yie Yang, ${ }^{* \dagger}{ }^{\dagger}$ James G. Phillips, ${ }_{\S}$ Jeanne A. Stuckey, ${ }_{\S}^{\S, \ddagger}$ Longchuan Bai, ${ }^{\dagger}$ Haiying Sun, ${ }^{\perp}$ James Delproposto, ${ }^{\S}$ William Clay Brown, ${ }^{\S}$ and Krishnapriya Chinnaswamy ${ }^{\S}$

\author{
${ }^{\dagger}$ Departments of Internal Medicine and ${ }^{\ddagger}$ Biological Chemistry and ${ }^{\S}$ Life Sciences Institute, University of Michigan, Ann Arbor, \\ Michigan 48109, United States \\ "Department of Translational Hematology and Oncology Research, Taussig Cancer Institute, Cleveland Clinic, Cleveland, Ohio \\ 44195, United States \\ ${ }^{\perp}$ Jiansu Key Laboratory of Drug Design and Optimization, Department of Medicinal Chemistry, School of Pharmacy, China \\ Pharmaceutical University, Nanjing 210009, China
}

\section{Supporting Information}

\begin{abstract}
Aberrant activation of the complement system is associated with diseases, including paroxysmal nocturnal hemoglobinuria and age-related macular degeneration. Complement factor $\mathrm{D}$ is the rate-limiting enzyme for activating the alternative pathway in the complement system. Recent development led to a class of potent amide containing pyrrolidine derived factor $\mathrm{D}$ inhibitors. Here, we used biochemical enzymatic and biolayer interferometry assays to demonstrate that the amide group improves the inhibitor potency by more than 80 -fold. Our crystal structures revealed

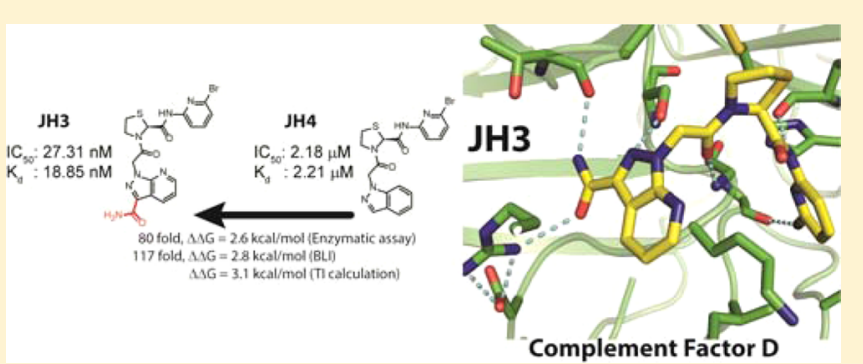
buried hydrogen bond interactions are important. Molecular orbital analysis from quantum chemistry calculations dissects the chemical groups participating in these interactions. Free energy calculation supports the differential contributions of the amide group to the binding affinities of these inhibitors. Cell-based hemolysis assay confirmed these compounds inhibit factor D mediated complement activation via the alternative pathway. Our study highlights the important interactions contributing to the high potency of factor D inhibitors reported recently.

KEYWORDS: Paroxysmal nocturnal hemoglobinuria, age-related macular degeneration, complement system, complement factor D inhibitors, serine protease, enzyme inhibition assay, biolayer interferometry assay, crystal structure, quantum chemistry calculations, thermodynamic integration method, computational docking, molecular dynamics simulations, hemolysis assay
\end{abstract}

$\mathrm{T}$ he complement system plays important roles as a first line of defense against microbial infection and in the clearance of injured tissues. The efficient activation of the complement system "complements" the relatively slower adaptive immune response to serve as a functional bridge between the innate and adaptive immune system. ${ }^{1}$ The complement system activation is known to be mediated by three known pathways, including classical pathway (CP, triggered by antigen-antibody complex formation), lectin pathway (LP, mediated by lectin binding to pathogen surface), and alternative pathway ( $\mathrm{AP}$, initiated by recognition to the activating pathogenic surface), which lead to inflammation, cell lysis, and opsonization. Through a cascade of proteolytic cleavage and activation, these pathways recruit a group of plasma proteins to assemble a membrane attack complex $(\mathrm{MAC})^{2}$ on the targeted cell surface which forms a hole in the membrane and causes cell lysis. Uncontrolled complement activation causes disease manifestations including paroxysmal nocturnal hemoglobinuria $(\mathrm{PNH})$, age-related macular degeneration, and neurodegenerative diseases. ${ }^{1,3,4}$
Among them, PNH is an acquired clonal disease of hematopoietic stem cells due to somatic mutation in the PIG-A gene. ${ }^{5}$ Mutated PIG-A protein causes deficient biosynthesis of glycophosphatidylinositol (GPI)-anchored surface proteins on the progeny blood cells. ${ }^{6}$ The GPI-linked CD55 and CD59 deficient erythrocytes lose protection from C5- and C3-involved complement activation, ${ }^{3,7}$ which triggers intravascular hemolysis. Activated complement system targeting the PIG-A mutant blood cells is also responsible for the cardinal symptoms of the disease, including hemolysis, thrombosis, and bone marrow failure observed in the $\mathrm{PNH}$ patients. ${ }^{8}$ Only one C5 antibody-based complement-targeted therapy (eculizumab) has been approved to treat PNH disease. ${ }^{9}$ Although eculizumab ameliorates anemia and reduces transfusion-dependence in PNH patients, a recent genetic study showed Japanese patients

Received: August 1, 2016

Accepted: September 13, 2016

Published: September 13, 2016 
with C5 variants are resistant to eculizumab treatment. ${ }^{10}$ Recently, a peptide-based inhibitor (AMY-101) $)^{3,7}$ targeting upstream C3 protein has received orphan drug status from US FDA and European Medicines Agency (EMA) for treating C3 glomerulopathy.

Several enzymes play important roles in complement activation by processing the complement proteins. Among them, the plasma concentration of complement factor D (denoted as factor D) was reported to be the lowest among all complement related proteins and considered a rate-limiting enzyme. Acting on the upstream of $\mathrm{AP}$, factor $\mathrm{D}$ cleaves complement factor $\mathrm{B}$ bound with $\mathrm{C} 3 \mathrm{~b}$ into the $\mathrm{C} 3 \mathrm{bBb}$ complex. ${ }^{11}$ The ubiquitous role of factor $\mathrm{D}$ in AP activation led to the implication that inhibition of factor $\mathrm{D}$ can be an attractive strategy in complement-targeted therapy. Factor D belongs to the serine protease family. ${ }^{12}$ Previous factor D inhibitor developments have led to the discovery of nonspecific catalytic site inhibitors, including coumarin derived covalent inhibitors $^{13,14}$ and the noncovalent inhibitor (compound 53) with $\mathrm{K}_{\mathrm{i}}=3.7 \mu \mathrm{M}^{15}$ (Figures 1A, S1). An antibody (AFD)

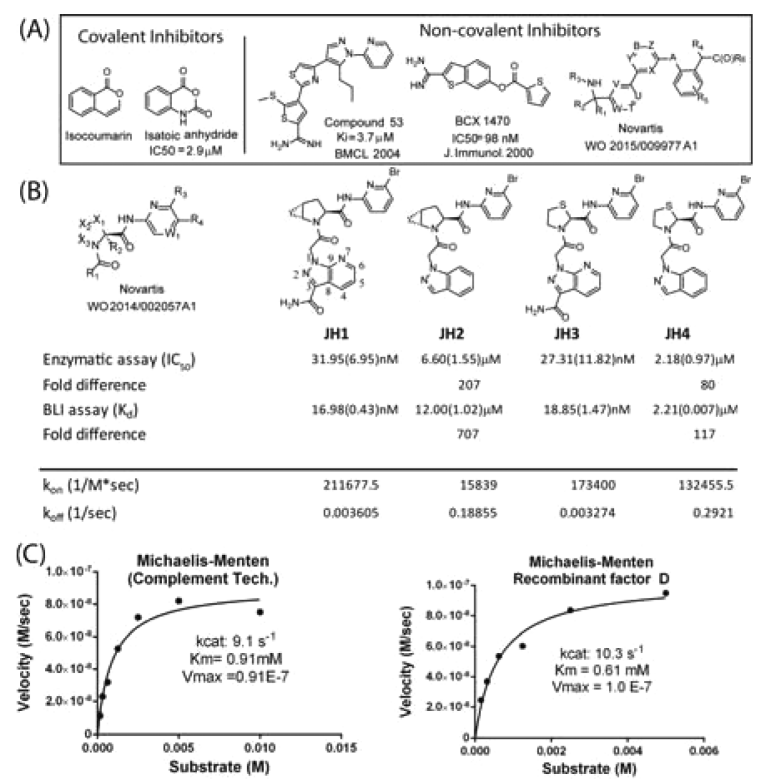

Figure 1. (A) Examples of the structures of the covalent and noncovalent factor D inhibitors. (B) Structures of JH1-JH4, their $\mathrm{IC}_{50}$, and binding kinetic data with factor D. For the enzymatic assay, the $\mathrm{IC}_{50}$ values were determined by the average of at least two independent triplicate experiments. Binding kinetic data were obtained from the averages of two independent experiments, and the standard deviations are shown in the parentheses. (C) Enzymatic activity parameters ${ }^{24}$ of factor D obtained from Complement Technology and our recombinant protein as determined from the enzymatic assay.

targeting an exosite in factor $\mathrm{D}$ has also been developed to block the binding between factor $\mathrm{D}$ and $\mathrm{C} 3 \mathrm{bB}$, with an $\mathrm{IC}_{50}$ value of $430 \mathrm{nM}^{16}$ Recently, aurin tricarboxylic acid (ATA), a promiscuous inhibitor that is prone to polymerization, ${ }^{17}$ was reported to bind to a noncatalytic site in factor $\mathrm{D}^{18}$ and inhibited the complement activation. These inhibitors lacking specificity and adequate potency have limited use in studying the consequence of specific factor $\mathrm{D}$ inhibition in disease models.

Recently, Novartis reported two classes of potent noncovalent factor $\mathrm{D}$ inhibitors based on the pyrrolidine and the aminomethyl-biaryl core structures (Figures 1A, S1) to treat age-related macular degeneration and diabetic retinopathy. ${ }^{19,20}$ Further, patents filed by Achillion pharmaceuticals (WO 2015/ 130784 A1, WO 2015/130795 A1, WO 2015/130806 A1, WO 2015/130830 A1, WO 2015/130838 A1, WO 2015/130842 A2, WO 2015/130845 A1, and WO 2015/130854 A1) focused on extensive optimization of the derivatives of a similar pyrrolidine core as factor D inhibitors. In the Novartis patent, ${ }^{19}$ an amide group coupled to the pyrazolopyridine group appears in multiple compounds but its contribution to the inhibitory activity is not clear nor claimed (see Figure 1). The amide group is not known to be a critical group for serine protease inhibitors reported previously. ${ }^{21}$ To elucidate the role of this chemical group, we combined chemical synthesis, in vitro biochemical and cell-based assays, crystal structure determination, and computational calculations to provide detailed insights into the interaction between factor $\mathrm{D}$ and the new class of inhibitors.

We first evaluated the enzymatic inhibition activity of four compounds (JH1-JH4 in Figure 1) based on two related core scaffolds using a well-known substrate, Z-Lys-thiobenzyl ester (Z-Lys-Sbzl). JH1 and JH3 were included in the Novartis patent. ${ }^{19}$ Factor D extracted from normal human serum purchased from Complement Technology was used in the assay. Our enzymatic assay showed the $\mathrm{IC}_{50}$ values for JH1-4 are $31.95 \mathrm{nM}, 6.60 \mu \mathrm{M}, 27.31 \mathrm{nM}$, and $2.18 \mu \mathrm{M}$, respectively. The data showed a 207 -fold change in $\mathrm{IC}_{50}$ values between $\mathrm{JH} 1$ and JH2. Using a different thiazolidine core, we found a lesser yet significant 80 -fold change in $\mathrm{IC}_{50}$ values between $\mathrm{JH} 3$ and JH4. The amide group indeed plays an important role in the inhibitory activity of these inhibitors.

We next determined the direct binding constants of the inhibitors to factor D by employing Biolayer interferometry (BLI) experiments. Because a larger amount of biotinylated proteins are required in this assay, recombinant human factor D expressed from Sf9 insect cells was used in the BLI experiments. The insect cell expression system has also been reported to produce matured human factor D previously. ${ }^{22}$ To confirm the enzymatic activity of our recombinant factor $\mathrm{D}$, we determined and compared the $K_{\mathrm{m}}, V_{\max }$ and $k_{\text {cat }}$ of our recombinant factor $\mathrm{D}$ with those using the factor $\mathrm{D}$ purchased from Complement Technology. As shown in Figure 1C, both factor D proteins gave similar $V_{\max }, K_{\mathrm{m}}$, and $k_{\text {cat }}$ using Z-LysSbzl as the substrate when the data were fitted to the Michaelis-Menten equation. Our $K_{\mathrm{m}}$ and $k_{\text {cat }}$ values are comparable to the previously reported $k_{\text {cat }}=1.30 \mathrm{~s}^{-1}$ and $K_{\mathrm{m}}$ $=3.79 \mathrm{mM}$ using the wild-type human factor $\mathrm{D}^{23}$ despite the differences of DMSO concentrations ( $4 \%$ versus $9 \%{ }^{23}$ ). We also tested the inhibition activity of $\mathrm{JH} 3$ to the recombinant factor D and obtained an $\mathrm{IC}_{50}$ value of $19 \mathrm{nM}$ (Figure S2). We concluded that our recombinant factor $\mathrm{D}$ has similar activity as the factor D purchased from Complement Technology.

Based on the BLI experiments, we observed that the dissociation constants, $K_{\mathrm{d}}$, between $\mathrm{JH1}-4$ and factor D are $16.98 \mathrm{nM}, 12.00 \mu \mathrm{M}, 18.85 \mathrm{nM}$, and $2.21 \mu \mathrm{M}$, respectively (Figure 1). The $K_{\mathrm{d}}$ values are consistent with and close to the $\mathrm{IC}_{50}$ values measured using the biochemical enzymatic inhibition assay. Comparison of the $K_{d}$ values indicated that JH2 is weaker than $\mathrm{JH1}$ by 707 -fold whereas $\mathrm{JH} 4$ is weaker than JH3 by 117 -fold. Data from two biochemical assays concluded that the contribution of the amide group to the inhibitors activities is more profound when the $(1 R, 5 S)-2$ azabicyclo[3.1.0] hexane core is used. Despite the differences of the cores, JH1 and $\mathrm{JH} 3$ have similar potencies to factor $\mathrm{D}$. 
Further analysis of the BLI data indicated that $k_{\text {off }}$ and $k_{\text {on }}$ of JH1 are similar to those of $\mathrm{JH} 3(0.003605$ versus $0.0032741 / \mathrm{s}$ and 211677.5 versus $173400.01 / \mathrm{Ms}$ ). Differences in the core structures of $\mathrm{JH} 1$ and $\mathrm{JH} 3$ have little influence on their binding kinetics with factor $\mathrm{D}$. When comparing $\mathrm{JH} 1$ with $\mathrm{JH} 2$ versus $\mathrm{JH} 3$ with $\mathbf{J H} 4$, the greater differential factor to their affinities is their $k_{\text {on }}$ values. The ratio of the $k_{\text {on }}$ values for $\mathrm{JH} 1$ and $\mathrm{JH} 2$ is 13 , which is much larger than the ratio of 1.3 between $\mathrm{JH} 3$ and JH4. In contrast, the ratio of $k_{\text {off }}$ for $\mathrm{JH} 1$ and $\mathrm{JH} 2$ is 52 , which is similar to the ratio of 89 between $\mathrm{JH} 3$ and JH4.

To gain structural insights, we obtained cocrystal structures of $\mathrm{JH} 3$ and $\mathrm{JH} 4$ with factor D at 3.15 and $3.36 \AA$ resolution, respectively. The electron density at the binding site was high enough to allow determination of the atomic positions of both inhibitors and interpretation of their interactions with factor D (see Figure 2). Figure 2 showed that a carbonyl group of $\mathbf{J H 1}$

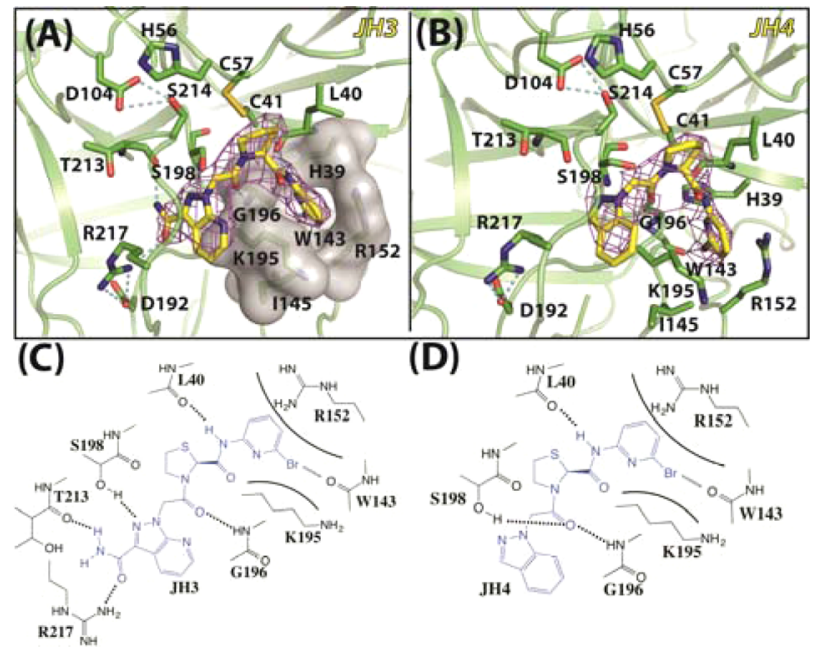

Figure 2. JH3 (A) and JH4 (B) are colored in yellow while factor D is colored in green. The mesh maps correspond to the $3 \sigma$ level differences when the electron density maps were fitted to factor D without the inhibitors. Hydrogen bond interaction is shown as a cyan dashed line. The binding pockets formed by H39, W143, I145, R152, $\mathrm{K} 195$, and G196 are shown in a surface representation colored in gray. (C-D) Depiction of the hydrogen bond, the cation $-\pi$, and the halogen bond interactions between $\mathrm{JH} 3, \mathrm{JH} 4$, and factor $\mathrm{D}$ identified from the cocrystal structures.

or JH3 interacts with the backbone amide groups of G196 and S198, representing the typical oxyanion hole interaction found in serine proteases, ${ }^{21}$ and $\mathrm{H} 56$ in the catalytic triad also adopts the atypical conformation. ${ }^{14}$ Furthermore, the amide group forms buried hydrogen bonds with the side chain of R217, the backbone carbonyl group of T213, and the hydroxyl group of S198. The crystal structure also indicates that the guanidine group of R217 orients similarly to R217 found in the DICbound 3,4-dichloroisocoumarin(DIC)-bound factor D crystal structure (PDBID: $1 \mathrm{DIC}^{13}$ ). Besides the interactions involving the amide group, the bromine atom of the pyridine group in $\mathrm{JH} 3$ is at a distance of $3.5 \AA$ to the carbonyl group of W143, representing a halogen bond interaction between the electron deficient $\sigma$-hole of the bromine atom and the electron-rich oxygen atom of the carbonyl group. ${ }^{25}$ An additional cation $-\pi$ interaction can be identified between the pyridine group and R152. Alignment of both inhibitor-bound factor D structures showed little backbone structural changes in factor D, as the root-mean-square-deviation of the backbone atoms between them is $0.16 \AA$. The structures also indicated that the indazole group (without the amide group) in JH3 and JH4 orients similarly whereas the amide group in $\mathbf{J H} 3$ extends deeper into the binding site. Comparison of the structures between JH4 and DIC showed that an aromatic group at the binding site close to the catalytic S198 location is favored. However, polar amino acids, including R217 and T213 and potentially a mediating water molecule, establish a hydrophilic environment deeper in the binding site to interact with the amide group of the pyrazolo $[3,4-b]$ pyridine group in $\mathbf{J H} 3$, unknown previously (Figure 2).

To investigate the role of water molecules at the binding site unresolved in our cocrystal structures because of low resolution, we examined all available crystal structures of factor D. We found one water molecule interacting with R217 and T213 is resolved in multiple crystal structures (PDBIDs: 1DIC, 1BIO, 1FDP, 1DSU). Placement of this water molecule (Wat387 in the factor D-DIC cocrystal structure) in the binding site of factor D with $\mathrm{JH} 3$ showed that this water molecule can form hydrogen bonds with the amide group of $\mathrm{JH} 3$, the $\mathrm{N} \omega^{1}$ of $\mathrm{R} 217$, and the hydroxyl group of T213. Thus, Wat387 was included in our computational analysis.

To dissect the network of hydrogen bonds between the pyrazolo[3,4- $b]$ pyridine group and neighboring amino acids in factor $\mathrm{D}$, we performed the fragment population analysis based on the quantum chemistry calculations. First, we optimized a simplified model of the binding site represented by the side chain of R217, T213, the side chain of S198 (approximated by the isobutanol), and Wat387 extracted from the crystal structure (see Figure S4). To approximate the electric fields in protein, ${ }^{26}$ the extracted fragments were embedded in diethyl ether, which has a dielectric constant of 4.24 used by the polarizable continuum model. ${ }^{27}$ The optimized structure showed that the local crystal structure was mostly preserved despite a substantial change of the hydrophobic side chain of R217 due to the lack of backbone constraint (Figure S4).

In the fragment population analysis, Wat $387, \mathrm{~T} 213$, the side chain of S198, the side chain of R217, and JH3 were treated as fragments. The hydrogen bond interactions between fragments were further verified via visual inspection of molecular orbitals. After examining the 30 highest occupied molecular orbitals of the optimized structure, we identified four types of hydrogen bond interactions (see Figure 3). The first type of interaction is between the side chain of $\mathrm{R} 217$ and $\mathrm{JH} 3$ as seen in the molecule orbital 204 (MO204) and MO206 (Figure 3A). While JH3 contributes $79 \%$ of electrons to MO204, R217 and JH3 equally participate in MO206. The electron densities also revealed a long-range resonance contribution of the lone pair electron from N7 on JH3 to both molecular orbitals. The second type of interaction is between $\mathrm{S} 198$ and $\mathbf{J H} 3$, as seen in MO189 and MO190 in Figure 3B. Our analysis shows that JH3/MO107 engages S194/LUMO and S194/HOMO-2 to form MO189 and MO190, respectively. The third type of hydrogen bond interaction involving $\mathrm{T} 213, \mathrm{~S} 198$, and $\mathrm{JH} 3$ is shown in Figure 3C. In this case, T213/HOMO-1 and T213/ HOMO-2 interact with S198/HOMO and JH3/MO118 to form MO210 and MO211, respectively, where T213 appears to be a mediator between $\mathrm{S} 198$ and JH3. The fourth type of interaction is between T213/HOMO-1 and JH3/MO118, as shown in Figure 3D. The p-type orbital on the amide group of JH3/MO118 can be found to participate in multiple hydrogen bond interactions, as demonstrated in Figures 3C-3D. The third and fourth types of interactions appear to involve only the 


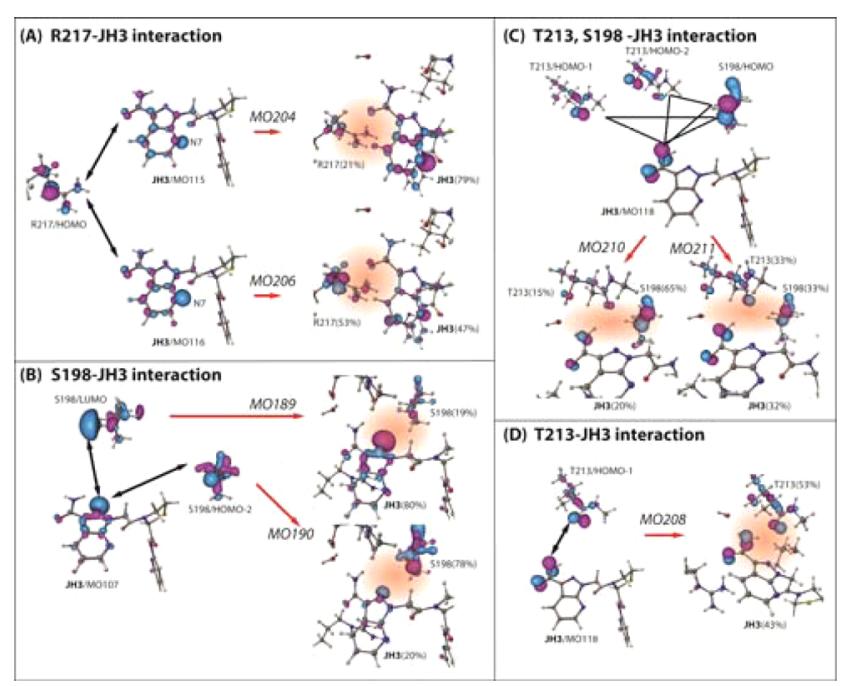

Figure 3. Molecular orbital analysis of four types of hydrogen bond interactions between the $1 \mathrm{H}$-pyrazolo[3,4-b] pyridine-3-carboxamide in JH3 and the neighboring residues. The percentage of electron density contributed from each fragment is shown in the parentheses. Energy levels of the molecular orbitals in reference to HOMO and LUMO are labeled with a plus and a minus number corresponding to the number of energy levels higher and lower than HOMO or LUMO.

amide group of JH3 whereas the electron delocalization in $\mathrm{JH} 3$ plays a prominent role in the first two types of interactions. Among the 30 highest occupied molecular orbitals analyzed, we found Wat 387 plays no role in hydrogen bonding. We conclude Wat387 does not contribute directly to the binding affinity of $\mathrm{JH} 3$ or $\mathrm{JH} 1$ with factor D.

Because no cocrystal structures between $\mathrm{JH1}, \mathrm{JH} 2$, and factor $\mathrm{D}$ were obtained, we modeled the binding poses of $\mathrm{JH1}$ and $\mathrm{JH} 2$ using the $\mathrm{JH} 3$-bound factor D structure (Figure S5) and computed the binding free energy changes of $\mathrm{JH1}-\mathrm{JH} 4$ via alchemical transformations to provide additional supporting evidence of their binding affinity differences. We first confirmed the stability of factor D in complex with $\mathrm{JH} 3$ and $\mathrm{JH} 4$ from the MD simulations (discussed in the SI) and calculated their binding free energy differences using the thermodynamic integration method. ${ }^{28}$ Between JH3 and JH4, our calculations gave a difference of $3.10 \pm 0.54 \mathrm{kcal} / \mathrm{mol}$ in $\Delta \Delta G$ (Table S2), which is comparable to the $2.82 \mathrm{kcal} / \mathrm{mol}$ estimated from BLI experiments. For $\mathrm{JH} 1$ and $\mathrm{JH} 2$, we obtained $\Delta \Delta G=5.63 \pm$ $0.20 \mathrm{kcal} / \mathrm{mol}$ (versus $3.89 \mathrm{kcal} / \mathrm{mol}$ from BLI), which is higher than that between $\mathrm{JH} 3$ and $\mathrm{JH} 4$. Thus, the binding free energy differences in $\mathbf{J H 1}-\mathbf{J H} 4$ obtained from our computational calculations are consistent with those calculated based on the $K_{\mathrm{d}}$ values of the BLI experiments and the $\mathrm{IC}_{50}$ values from the enzymatic assay.

To demonstrate these compounds inhibit complement activation by targeting factor $\mathrm{D}$ in $\mathrm{AP}$, we evaluated them in the standard rabbit erythrocyte hemolysis assay. Activation of $\mathrm{AP}$ was induced by introducing human serum to rabbit erythrocytes in the presence of $\mathrm{Mg}^{2+}$ while depleting $\mathrm{Ca}^{2+}$ ions via EGTA. Figure 4 showed the most potent inhibitor to the hemolysis is $\mathbf{J H} 1$ followed by $\mathbf{J H} 3$. The $\mathrm{IC}_{50}$ values of $\mathrm{JH} 2$ and $\mathrm{JH} 4$ are at micromolar ranges $(77$ and $19 \mu \mathrm{M})$. Although $\mathrm{JH} 3$ has similar enzymatic $\mathrm{IC}_{50}$ and $K_{\mathrm{d}}$ values as $\mathrm{JH1}$, yet the $\mathrm{IC}_{50}$ value of JH3 (205 nM) observed in the hemolysis assay is 4fold higher than that of JH1 (47 nM) (Figure 4). Whether serum protein binding plays a role to decrease the free JH3

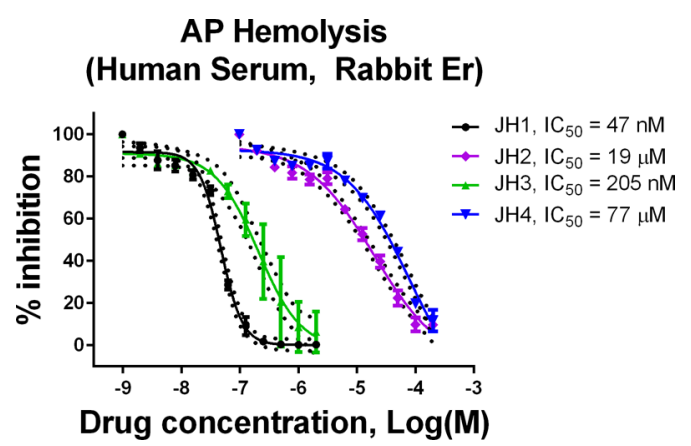

Figure 4. Inhibition of factor $\mathrm{D}$ by JH1-4 in the hemolysis assay using the normal human serum and rabbit erythrocytes. The $\mathrm{IC}_{50}$ values are shown as the mean \pm SE from triplicate $(n=3)$ experiments.

concentration leading to the reduced $\mathrm{IC}_{50}$ value in the hemolysis assay remains to be determined.

In summary, our data, including the binding between four inhibitors and factor D obtained from in vitro biochemical assays, crystal structures, computational calculations, and the cell-based hemolysis assay, reveal the critical role of the amide group in these inhibitors for their high potencies to factor D. The findings can be useful for developing potent and selective complement factor D inhibitors in the future to assess their therapeutic potential in disease models.

\section{ASSOCIATED CONTENT}

\section{S Supporting Information}

The Supporting Information is available free of charge on the ACS Publications website at DOI: 10.1021/acsmedchemlett.6b00299.

Experimental binding data, the docking models, and methods (PDF)

\section{AUTHOR INFORMATION}

\section{Corresponding Author}

*E-mail: chaoyie@umich.edu. Phone: (734) 764-2449.

\section{Author Contributions}

J.G.P. and H.S. synthesized the compounds; J.A.S. solved the crystal structures; C.-Y.Y. performed the enzymatic assay; J.D. conducted the BLI assay; L.B. performed the hemolysis assay; W.C.B. cloned and expressed the protein; K.C. purified the protein; C.-Y.Y. conducted computational calculations, designed the project, and wrote the manuscript; the manuscript was written through contributions of all authors.

\section{Funding}

We are grateful for financial support from the Aplastic Anemia MDS international Foundation and from the National Institutes of Health through the University of Michigan Cancer Center Support Grant (P30CA046592) by the use of the following Cancer Center Core: Center of Structural Biology. Use of the Advanced Photon Source was supported by the U.S. Department of Energy, Office of Science, Office of Basic Energy Sciences, under Contract No. DE-AC02-06CH11357. Use of the LS-CAT Sector 21 was supported by the Michigan Economic Development Corporation and the Michigan Technology Tri-Corridor for the support of this research program (Grant 085P1000817).

Notes

The authors declare no competing financial interest. 


\section{ACKNOWLEDGMENTS}

We thank Jaroslaw Maciejewski from Cleveland Clinic for introducing us to $\mathrm{PNH}$ and for helpful discussions, and Shaomeng Wang from University of Michigan for providing equipment to conduct enzymatic and hemolysis assays. We also thank Liu Liu, Bruce Palfey, and Mou-Chi Cheng for helpful discussions on the enzymatic assay.

\section{REFERENCES}

(1) Ricklin, D.; Hajishengallis, G.; Yang, K.; Lambris, J. D. Complement: a key system for immune surveillance and homeostasis. Nat. Immunol. 2010, 11, 785-797.

(2) Serna, M.; Giles, J. L.; Morgan, B. P.; Bubeck, D. Structural basis of complement membrane attack complex formation. Nat. Commun. 2016, 7, 10587.

(3) Mastellos, D. C.; Ricklin, D.; Yancopoulou, D.; Risitano, A.; Lambris, J. D. Complement in paroxysmal nocturnal hemoglobinuria: exploiting our current knowledge to improve the treatment landscape. Expert Rev. Hematol. 2014, 7, 583-598.

(4) Ricklin, D.; Lambris, J. D. Progress and trends in complement therapeutics. Adv. Exp. Med. Biol. 2013, 735, 1-22.

(5) Kinoshita, T.; Takeda, J. [GPI-anchored proteins and paroxysmal nocturnal hemoglobinuria]. Nippon Saikingaku Zasshi 1993, 48, 533539.

(6) Tomita, M. Biochemical background of paroxysmal nocturnal hemoglobinuria. Biochim. Biophys. Acta, Mol. Basis Dis. 1999, 1455, 269-286.

(7) Brodsky, R. A. Complement in hemolytic anemia. Blood 2015, 126, 2459-2465.

(8) Parker, C.; Omine, M.; Richards, S.; Nishimura, J.; Bessler, M.; Ware, R.; Hillmen, P.; Luzzatto, L.; Young, N.; Kinoshita, T.; Rosse, W.; Socie, G.; International, P. N. H. I. G. Diagnosis and management of paroxysmal nocturnal hemoglobinuria. Blood 2005, 106, 36993709.

(9) Rother, R. P.; Rollins, S. A.; Mojcik, C. F.; Brodsky, R. A.; Bell, L. Discovery and development of the complement inhibitor eculizumab for the treatment of paroxysmal nocturnal hemoglobinuria. Nat. Biotechnol. 2007, 25, 1256-1264.

(10) Nishimura, J.-i.; Yamamoto, M.; Hayashi, S.; Ohyashiki, K.; Ando, K.; Brodsky, A. L.; Noji, H.; Kitamura, K.; Eto, T.; Takahashi, T.; Masuko, M.; Matsumoto, T.; Wano, Y.; Shichishima, T.; Shibayama, H.; Hase, M.; Li, L.; Johnson, K.; Lazarowski, A.; Tamburini, P.; Inazawa, J.; Kinoshita, T.; Kanakura, Y. Genetic Variants in C5 and Poor Response to Eculizumab. N. Engl. J. Med. 2014, 370, 632-639.

(11) Forneris, F.; Ricklin, D.; Wu, J.; Tzekou, A.; Wallace, R. S.; Lambris, J. D.; Gros, P. Structures of C3b in complex with factors B and $\mathrm{D}$ give insight into complement convertase formation. Science 2010, 330, 1816-1820.

(12) Di Cera, E. Serine proteases. IUBMB Life 2009, 61, 510-515.

(13) Cole, L. B.; Kilpatrick, J. M.; Chu, N.; Babu, Y. S. Structure of 3,4-dichloroisocoumarin-inhibited factor D. Acta Crystallogr., Sect. D: Biol. Crystallogr. 1998, 54, 711-717.

(14) Jing, H.; Babu, Y. S.; Moore, D.; Kilpatrick, J. M.; Liu, X. Y.; Volanakis, J. E.; Narayana, S. V. Structures of native and complexed complement factor D: implications of the atypical His57 conformation and self-inhibitory loop in the regulation of specific serine protease activity. J. Mol. Biol. 1998, 282, 1061-1081.

(15) Subasinghe, N. L.; Ali, F.; Illig, C. R.; Jonathan Rudolph, M.; Klein, S.; Khalil, E.; Soll, R. M.; Bone, R. F.; Spurlino, J. C.; DesJarlais, R. L.; Crysler, C. S.; Cummings, M. D.; Morris, P. E., Jr.; Kilpatrick, J. M.; Sudhakara Babu, Y. A novel series of potent and selective small molecule inhibitors of the complement component C1s. Bioorg. Med. Chem. Lett. 2004, 14, 3043-3047.

(16) Katschke, K. J., Jr.; Wu, P.; Ganesan, R.; Kelley, R. F.; Mathieu, M. A.; Hass, P. E.; Murray, J.; Kirchhofer, D.; Wiesmann, C.; van Lookeren Campagne, M. Inhibiting alternative pathway complement activation by targeting the factor D exosite. J. Biol. Chem. 2012, 287, 12886-12892.

(17) Lee, M.; Guo, J. P.; Schwab, C.; McGeer, E. G.; McGeer, P. L. Selective inhibition of the membrane attack complex of complement by low molecular weight components of the aurin tricarboxylic acid synthetic complex. Neurobiol. Aging 2012, 33, 2237-2246.

(18) Lee, M.; Guo, J. P.; McGeer, E. G.; McGeer, P. L. Aurin tricarboxylic acid self-protects by inhibiting aberrant complement activation at the $\mathrm{C} 3$ convertase and $\mathrm{C} 9$ binding stages. Neurobiol. Aging 2013, 34, 1451-1461.

(19) Hommel, U.; Lorthiois, E. L. J.; Maibaum, J. K.; Ostermann, N.; Randl, S. A.; Vulpetti, A.; Rogel, O. Pyrrolidine derivatives and their use as complement pathway modulators. WO 2014/002057 A1, 2014.

(20) Belanger, D. B.; Flohr, S.; Gelin, C. F.; Jendza, K.; Ji, N.; LIU, D.; Lorthiois, E. L. J.; Karki, R. G.; Mainolfi, N.; Powers, J. J.; Randl, S. A.; Rogel, O.; Vulpetti, A.; Yoon, T. Aminomethyl-biaryl derivatives as complement factor D inhibitors and uses thereof. WO 2015/009977 A1, 2015.

(21) Hedstrom, L. Serine protease mechanism and specificity. Chem. Rev. 2002, 102, 4501-4524.

(22) Jing, H.; Macon, K. J.; Moore, D.; DeLucas, L. J.; Volanakis, J. E.; Narayana, S. V. Structural basis of profactor D activation: from a highly flexible zymogen to a novel self-inhibited serine protease, complement factor D. EMBO J. 1999, 18, 804-814.

(23) Kim, S.; Narayana, S. V.; Volanakis, J. E. Crystal structure of a complement factor D mutant expressing enhanced catalytic activity. J. Biol. Chem. 1995, 270, 24399-24405.

(24) Conversion of the OD value to the enzyme concentration was calculated using the extinction coefficient of $13260 \mathrm{M}^{-1} \mathrm{~cm}^{-1}$ for the catalyzed product and the $0.29 \mathrm{~cm}$ path correction for $100 \mu \mathrm{L}$ of solution in each well.

(25) Wilcken, R.; Zimmermann, M. O.; Lange, A.; Joerger, A. C.; Boeckler, F. M. Principles and applications of halogen bonding in medicinal chemistry and chemical biology. J. Med. Chem. 2013, 56, $1363-1388$.

(26) Kukic, P.; Farrell, D.; McIntosh, L. P.; García-Moreno E, B.; Jensen, K. S.; Toleikis, Z.; Teilum, K.; Nielsen, J. E. Protein Dielectric Constants Determined from NMR Chemical Shift Perturbations. J. Am. Chem. Soc. 2013, 135, 16968-16976.

(27) Tomasi, J.; Mennucci, B.; Cammi, R. Quantum mechanical continuum solvation models. Chem. Rev. 2005, 105, 2999-3093.

(28) Kollman, P. Free energy calculations: Applications to chemical and biochemical phenomena. Chem. Rev. 1993, 93, 2395-2417. 